\title{
Qualifier Origin
}

National Cancer Institute

\section{Source}

National Cancer Institute. Qualifier Origin. NCI Thesaurus. Code C83366.

The source of a data modifier. 